\title{
International consensus diagnostic criteria for neuromyelitis optica spectrum disorders
} OPEN

Dean M. Wingerchuk, $\mathrm{MD}, \mathrm{FRCP}(\mathrm{C})$

Brenda Banwell, MD, $\operatorname{FRCP}(\mathrm{C})$

Jeffrey L. Bennett, MD, $\mathrm{PhD}$

Philippe Cabre, MD

William Carroll, MD

Tanuja Chitnis, MD Jérôme de Seze, MD Kazuo Fujihara, MD Benjamin Greenberg, MD

Anu Jacob, MD

Sven Jarius, MD

Marco Lana-Peixoto, MD

Michael Levy, MD, PhD Jack H. Simon, MD Silvia Tenembaum, MD Anthony L. Traboulsee, $\mathrm{MD}, \mathrm{FRCP}(\mathrm{C})$ Patrick Waters, PhD Kay E. Wellik, MLS Brian G. Weinshenker, $\mathrm{MD}, \mathrm{FRCP}(\mathrm{C})$

Correspondence to Dr. Wingerchuk: wingerchuk.dean@mayo.edu or Dr. Weinshenker: weinb@mayo.edu

Editorial, page 118

Supplemental data at Neurology.org

\section{ABSTRACT}

Neuromyelitis optica (NMO) is an inflammatory CNS syndrome distinct from multiple sclerosis (MS) that is associated with serum aquaporin-4 immunoglobulin $G$ antibodies (AQP4-lgG). Prior NMO diagnostic criteria required optic nerve and spinal cord involvement but more restricted or more extensive CNS involvement may occur. The International Panel for NMO Diagnosis (IPND) was convened to develop revised diagnostic criteria using systematic literature reviews and electronic surveys to facilitate consensus. The new nomenclature defines the unifying term NMO spectrum disorders (NMOSD), which is stratified further by serologic testing (NMOSD with or without AQP4-lgG). The core clinical characteristics required for patients with NMOSD with AQP4-IgG include clinical syndromes or MRI findings related to optic nerve, spinal cord, area postrema, other brainstem, diencephalic, or cerebral presentations. More stringent clinical criteria, with additional neuroimaging findings, are required for diagnosis of NMOSD without AQP4IgG or when serologic testing is unavailable. The IPND also proposed validation strategies and achieved consensus on pediatric NMOSD diagnosis and the concepts of monophasic NMOSD and opticospinal MS. Neurology ${ }^{\circledR}$ 2015;85:177-189

\section{GLOSSARY}

ADEM = acute disseminated encephalomyelitis; AQP4 = aquaporin-4; IgG = immunoglobulin G; IPND = International Panel for NMO Diagnosis; LETM = longitudinally extensive transverse myelitis lesions; MOG = myelin oligodendrocyte glycoprotein; $\mathbf{M S}=$ multiple sclerosis; NMO = neuromyelitis optica; NMOSD = neuromyelitis optica spectrum disorders; SLE = systemic lupus erythematosus; $\mathbf{S S}=$ Sjögren syndrome.

Neuromyelitis optica (NMO) is an inflammatory CNS disorder distinct from multiple sclerosis (MS). ${ }^{1,2}$ It became known as Devic disease following a seminal 1894 report. ${ }^{3, \mathrm{e} 1, \mathrm{e} 2}$ Traditionally, NMO was considered a monophasic disorder consisting of simultaneous bilateral optic neuritis and transverse myelitis but relapsing cases were described in the 20th century. ${ }^{3}$ MRI revealed normal brain scans and $\geq 3$ vertebral segment longitudinally extensive transverse myelitis lesions (LETM) in NMO. ${ }^{4, e 3}$ The nosology of NMO, especially whether it represented a topographically restricted form of MS, remained controversial.

A major advance was the discovery that most patients with $\mathrm{NMO}$ have detectable serum antibodies that target the water channel aquaporin-4 (AQP4-immunoglobulin G [IgG]), 5,6 are highly specific for clinically diagnosed NMO, and have pathogenic potential. ${ }^{7, \mathrm{e} 4 \mathrm{e} 6}$ In 2006, AQP4-IgG serology was incorporated into revised NMO diagnostic criteria that relaxed clinical

\footnotetext{
From the Departments of Neurology (D.M.W.) and Library Services (K.E.W.), Mayo Clinic, Scottsdale, AZ; the Children's Hospital of Philadelphia (B.B.), PA; the Departments of Neurology and Ophthalmology (J.L.B.), University of Colorado Denver, Aurora; the Service de Neurologie (P.C.), Centre Hospitalier Universitaire de Fort de France, Fort-de-France, Martinique; Department of Neurology (W.C.), Sir Charles Gairdner Hospital, Perth, Australia; the Department of Neurology (T.C.), Massachusetts General Hospital, Boston; the Department of Neurology (J.d.S.), Strasbourg University, France; the Department of Multiple Sclerosis Therapeutics (K.F.), Tohoku University Graduate School of Medicine, Sendai, Japan; the Departments of Neurology and Neurotherapeutics (B.G.), University of Texas Southwestern Medical Center, Dallas; The Walton Centre NHS Trust (A.J.), Liverpool, UK; the Molecular Neuroimmunology Group, Department of Neurology (S.J.), University Hospital Heidelberg, Germany; the Center for Multiple Sclerosis Investigation (M.L.-P.), Federal University of Minas Gerais Medical School, Belo Horizonte, Brazil; the Department of Neurology (M.L.), Johns Hopkins University, Baltimore, MD; Portland VA Medical Center and Oregon Health and Sciences University (J.H.S.), Portland; the Department of Neurology (S.T.), National Pediatric Hospital Dr. Juan P. Garrahan, Buenos Aires, Argentina; the Department of Medicine (A.L.T.), University of British Columbia, Vancouver, Canada; Nuffield Department of Clinical Neurosciences (P.W.), University of Oxford, UK; and the Department of Neurology (B.G.W.), Mayo Clinic, Rochester, MN.

Go to Neurology.org for full disclosures. Funding information and disclosures deemed relevant by the authors, if any, are provided at the end of the article. The Article Processing Charge was paid by the Guthy-Jackson Charitable Foundation.

This is an open access article distributed under the terms of the Creative Commons Attribution-NonCommercial-NoDerivatives License 4.0 (CC BY-NC-ND), which permits downloading and sharing the work provided it is properly cited. The work cannot be changed in any way or used commercially.
} 
requirements by permitting unilateral optic neuritis or asymptomatic brain MRI lesions but retained the requirement for both myelitis and optic neuritis. ${ }^{2}$ The 2006 criteria were validated in several different ethnic and racial cohorts worldwide and became the standard for clinical and research purposes. ${ }^{8-10, e 5, e 7-\mathrm{e} 15}$

The specificity of AQP4-IgG facilitated observations that further broadened the clinical and neuroimaging spectrum of NMO. In 2007, the term NMO spectrum disorders (NMOSD) was introduced to include AQP4IgG-seropositive patients with limited or inaugural forms of NMO (e.g., first-attack LETM or recurrent or bilateral optic neuritis) who were at high risk for future attacks. ${ }^{1}$ The NMOSD term also encompassed the cerebral, diencephalic, and brainstem lesions that occur in a minority of patients with otherwise typical NMO. It also included AQP4-IgG-seropositive patients with coexisting autoimmune disorders (e.g., systemic lupus erythematosus [SLE] or Sjögren syndrome [SS]). Finally, NMOSD potentially included patients diagnosed with opticospinal MS, an MS phenotype prominent in Asia and distinguished from Western MS. ${ }^{11}$

Further advances have rendered the 2006 criteria inadequate for contemporary practice and research. Improvement in AQP4-IgG sensitivity has allowed for refinement of the list of nonopticospinal disease characteristics. ${ }^{12-14, \mathrm{e} 16-\mathrm{e} 18}$ Moreover, loss of AQP4 immunoreactivity and astrocyte pathology in brain and spinal cord NMO lesions distinguish them from MS lesions. ${ }^{\text {e19-e23 }}$ Together, these data suggest that non-opticospinal clinical and MRI characteristics should be incorporated into the diagnostic criteria. The term NMOSD has also been used variably in the literature and needs clarification. ${ }^{3}$ Other outstanding issues include whether there are distinctive features of pediatric NMO, the current value of the term opticospinal MS, and whether monophasic NMO can be defined. Finally, treatment strategies for attack prevention in NMO and MS differ. Some MS immunotherapies appear to aggravate NMO, indicating an imperative for early, accurate diagnosis. ${ }^{15-18, e 24-e 26}$ The International Panel for NMO Diagnosis (IPND) was convened and charged with revising NMO diagnostic criteria for clinical decision-making and to address the ancillary issues outlined above. This report represents the Panel's consensus recommendations.

METHODS The IPND consisted of 18 members from 9 countries and was led by 2 co-chairs (D.M.W., B.G.W.). It convened 7 times between October 2011 and November 2013. Panel members participated in 6 Working Groups: Clinical Presentation, Neuroimaging, Laboratory Studies/Serology, Pediatrics, Systemic Autoimmunity, and Opticospinal MS.

Initial consensus was reached on 2 points. First, NMO would be subsumed into the single descriptive term NMOSD because the clinical behavior, immunopathogenesis, and treatment of patients who have NMOSD are not demonstrably different than for those with NMO and patients with incomplete forms of NMO frequently later fulfill NMO criteria. ${ }^{1,14,19,20,227}$ The entrenched and historically significant phrase NMO would be retained by using NMOSD because, although the criteria would be broadened to include patients with neither optic neuritis or myelitis, ${ }^{21}$ those syndromes eventually occur in almost all patients. Second, revised criteria would define a clinical diagnosis by integrating clinical, serologic, and neuroimaging data; diagnosis would not be based solely on detection of AQP4-IgG. Moreover, the Panel concluded that criteria must define NMOSD in instances where AQP4-IgG serologic testing is negative or unavailable, especially because of the treatment implications.

The IPND established several goals, chief of which was developing revised consensus NMOSD criteria using the best available evidence. The Clinical Presentation Working Group conducted the primary task of establishing the spectrum and validity of clinical syndromes described in clinically diagnosed NMO or in association with AQP4-IgG; these data were used to establish new core clinical criteria. The other Working Groups each addressed a roster of focused questions that would contribute to final diagnostic criteria.

Each Working Group conducted a systematic literature review, with expert librarian assistance, to address its assignments. Medical subject heading terms were used when possible and MEDLINE, EMBASE, and other databases were searched from 1946-January 2012 with quarterly updates through January 15, 2014 (see table e-1 on the Neurology ${ }^{\circledR}$ Web site at Neurology.org for search strategies and results). Two individuals independently reviewed abstracts for relevance; discordant ratings were resolved by consensus. Relevant full-text articles were independently rated to identify those used for data extraction. Publications describing sensitivity and specificity of AQP4-IgG were included if they compared groups diagnosed with clinical NMO and MS.

The Clinical Presentation and Neuroimaging Working Groups compiled a list of clinical syndromes and MRI characteristics reported by more than one publication to be associated with NMOSD or AQP4-IgG. Panel members rated the specificity of these characteristics for NMOSD diagnosis using 2 electronic surveys. Two additional surveys presented adult case vignettes that contained information on clinical symptoms and signs (1-3 discrete clinical events) with brain MRI, AQP4-IgG serostatus, and other potential supportive criteria (optic nerve or spinal cord MRI findings) or laboratory results (e.g., CSF data, visual evoked potentials). Panel members assigned a diagnosis for each vignette: definite NMOSD, indeterminate (requiring further data or follow-up for confident diagnosis), or other (e.g., MS). A roster of potential supportive clinical, MRI, and laboratory criteria was 
separately ranked for their influence on confidence in NMOSD diagnosis. Characteristics or scenarios endorsed by a two-thirds Panel majority as contributing to a confident clinical diagnosis of NMOSD were used to develop separate requirements for AQP4IgG seropositive and seronegative patients. Recommendations from individual Working Groups regarding pediatric NMOSD, opticospinal MS, systemic autoimmunity, and monophasic disease were summarized. All IPND members endorsed the final criteria.

RESULTS Consensus diagnostic criteria. Nomenclature. The Panel reaffirmed the decision to unify the terms NMO and NMOSD. Given the greater degree of diagnostic uncertainty and potential heterogeneity of seronegative NMOSD, criteria were developed for both NMOSD with AQP4-IgG and NMOSD without AQP4-IgG. An additional category of NMOSD with unknown AQP4-IgG status may be used for patients in whom serologic testing is unavailable. The nomenclature allows for future modifications based on potential discovery and validation of other biomarkers in AQP4-IgG-seronegative patients who have otherwise typical NMOSD clinical syndromes.

Clinical presentation. Consensus diagnostic criteria for NMOSD are presented in table 1. Table e- 2 contains a glossary of terms to guide interpretation of individual components of the criteria. The criteria allow NMOSD diagnosis with occurrence of at least 1 of 6 core clinical characteristics and detection of AQP4-IgG. The core

\section{Table 1 NMOSD diagnostic criteria for adult patients}

Diagnostic criteria for NMOSD with AQP4-IgG

1. At least 1 core clinical characteristic

2. Positive test for AQP4-IgG using best available detection method (cell-based assay strongly recommended)

3. Exclusion of alternative diagnoses ${ }^{a}$

Diagnostic criteria for NMOSD without AOP4-lgG or NMOSD with unknown AOP4-IgG status

1. At least 2 core clinical characteristics occurring as a result of one or more clinical attacks and meeting all of the following requirements:

a. At least 1 core clinical characteristic must be optic neuritis, acute myelitis with LETM, or area postrema syndrome

b. Dissemination in space ( 2 or more different core clinical characteristics)

c. Fulfillment of additional MRI requirements, as applicable

2. Negative tests for AQP4-IgG using best available detection method, or testing unavailable

3. Exclusion of alternative diagnoses ${ }^{\mathrm{a}}$

Core clinical characteristics

1. Optic neuritis

2. Acute myelitis

3. Area postrema syndrome: episode of otherwise unexplained hiccups or nausea and vomiting

4. Acute brainstem syndrome

5. Symptomatic narcolepsy or acute diencephalic clinical syndrome with NMOSD-typical diencephalic MRI lesions (figure 3)

6. Symptomatic cerebral syndrome with NMOSD-typical brain lesions (figure 3)

Additional MRI requirements for NMOSD without AQP4-IgG and NMOSD with unknown

AQP4-lgG status

1. Acute optic neuritis: requires brain MRI showing (a) normal findings or only nonspecific white matter lesions, OR (b) optic nerve MRI with T2-hyperintense lesion or T1-weighted gadoliniumenhancing lesion extending over $>1 / 2$ optic nerve length or involving optic chiasm (figure 1)

2. Acute myelitis: requires associated intramedullary MRI lesion extending over $\geq 3$ contiguous segments (LETM) OR $\geq 3$ contiguous segments of focal spinal cord atrophy in patients with history compatible with acute myelitis (figure 1)

3. Area postrema syndrome: requires associated dorsal medulla/area postrema lesions (figure 2)

4. Acute brainstem syndrome: requires associated periependymal brainstem lesions (figure 2)

Abbreviations: AQP4 = aquaporin-4; IgG = immunoglobulin G; LETM = longitudinally extensive transverse myelitis lesions; NMOSD = neuromyelitis optica spectrum disorders.

a See table 2 and text discussion on serologic considerations for recommendations regarding interpretation of clinical and serologic testing. clinical characteristics each implicate 1 of 6 CNS regions: optic nerve, ${ }^{19,228-e 31}$ spinal cord, ${ }^{20, e 27, e 32-e 34}$ area postrema of the dorsal medulla, ${ }^{22,23, e 35}$ brainstem, ${ }^{24,35-e 38}$ diencephalon, ${ }^{25,26, e 39-e 44}$ or cerebrum. ${ }^{27,28, e 45-e 48}$ Certain clinical presentations are particularly suggestive of NMOSD: optic neuritis that is simultaneously bilateral, involves the optic chiasm, causes an altitudinal visual field defect, or causes severe residual visual loss (acuity 20/200 or worse $)^{4, e 49-e 51}$; a complete (rather than partial) spinal cord syndrome, especially with paroxysmal tonic spasms $s^{4,29, \mathrm{e} 52}$; and an area postrema clinical syndrome (16\%-43\% incidence) consisting of intractable hiccups or nausea and vomiting. ${ }^{22,23, e 35}$ Clinical judgment remains necessary because no characteristic is pathognomonic. For example, altitudinal visual field defects may result from ischemic optic neuropathy and bilateral simultaneous optic neuritis may occur in MS. ${ }^{\text {553 }}$

Diagnostic requirements are more stringent for patients in whom AQP4-IgG is not detected or for whom testing is unavailable. Such individuals must experience 2 or more different core clinical characteristics (i.e., dissemination in space, affecting different neuroanatomic regions) and other supportive MRI characteristics meant to enhance diagnostic specificity must also be present. At least one of the clinical events must be one of the 3 most common clinical characteristics of NMOSD: optic neuritis, transverse myelitis (additional requirement: LETM MRI lesion), or an area postrema clinical syndrome (additional requirement: associated medullary MRI lesion). ${ }^{22,23,30, e 35}$ The 2 required core characteristics may occur with a single clinical attack (e.g., classic Devic syndrome with simultaneous optic neuritis and acute myelitis with LETM) or multiple attacks.

The Panel reached several additional conclusions. First, at least 1 discrete clinical attack of CNS symptoms must occur to establish NMOSD diagnosis. Although asymptomatic AQP4-IgG seropositive status may exist for years before clinical NMOSD presentation, ${ }^{\text {e54 }}$ the natural history of asymptomatic seropositivity is poorly understood. Second, NMOSD diagnosis is not warranted in asymptomatic patients with NMOSD-compatible MRI lesions because the expected clinical course in such individuals is unknown. Third, no clinical characteristic is pathognomonic of NMOSD. Accordingly, a single clinical manifestation is not diagnostic when AQP4-IgG is not detected. Finally, no single characteristic is exclusionary but some are considered red flags (table 2) that signal the possibility of alternative diagnoses. .55-e63 $^{-6}$ The main clinical red flags concern the temporal course of the syndrome rather than the actual manifestations. Most notably, a gradually progressive course of neurologic worsening over months to years is very uncommon $(1 \%-2 \%)$ in NMOSD. ${ }^{31}$ However, after 
thorough investigation for potential competing disorders, the weight of evidence may justify NMOSD diagnosis despite presence of 1 or more red flags. The presence of systemic autoimmune diseases and certain CSF data and pathologic findings (see below) may also influence NMOSD likelihood. Regardless of AQP4-IgG serologic status, NMOSD should be diagnosed when criteria are fulfilled and alternative diagnoses for the clinical syndrome have been excluded.

Neuroimaging and neurophysiologic testing. MRI lesion patterns are a major arbiter of CNS demyelinating disease differential diagnosis. Several brain, optic nerve, and spinal cord patterns are characteristic or highly suggestive of NMOSD (table 3; figures 1-3). Detection of a LETM spinal cord lesion (figure 1)

\section{Table 2 Red flags: Findings atypical for NMOSD}

\section{Red flags (clinical/laboratory)}

1. Clinical features and laboratory findings

Progressive overall clinical course (neurologic deterioration unrelated to attacks; consider MS)

Atypical time to attack nadir: less than 4 hours (consider cord ischemia/infarction); continual worsening for more than 4 weeks from attack onset (consider sarcoidosis or neoplasm)

Partial transverse myelitis, especially when not associated with LETM MRI lesion (consider MS)

Presence of CSF oligoclonal bands (oligoclonal bands occur in $<20 \%$ of cases of NMO vs $>80 \%$ of MS)

2. Comorbidities associated with neurologic syndromes that mimic NMOSD

Sarcoidosis, established or suggestive clinical, radiologic, or laboratory findings thereof (e.g., mediastinal adenopathy, fever and night sweats, elevated serum angiotensin converting enzyme or interleukin-2 receptor levels)

Cancer, established or with suggestive clinical, radiologic, or laboratory findings thereof: consider lymphoma or paraneoplastic disease (e.g., collapsin response mediator protein-5 associated optic neuropathy and myelopathy or anti-Ma-associated diencephalic syndrome)

Chronic infection, established or with suggestive clinical, radiologic, or laboratory findings thereof (e.g., HIV, syphilis)

\section{Red flags (conventional neuroimaging)}

1. Brain

a. Imaging features (T2-weighted MRI) suggestive of MS (MS-typical)

Lesions with orientation perpendicular to a lateral ventricular surface (Dawson fingers) Lesions adjacent to lateral ventricle in the inferior temporal lobe

Juxtacortical lesions involving subcortical U-fibers

Cortical lesions

b. Imaging characteristics suggestive of diseases other than MS and NMOSD

Lesions with persistent $(>3 \mathrm{mo}$ ) gadolinium enhancement

2. Spinal cord

Characteristics more suggestive of MS than NMOSD

Lesions $<3$ complete vertebral segments on sagittal T2-weighted sequences

Lesions located predominantly (>70\%) in the peripheral cord on axial T2-weighted sequences

Diffuse, indistinct signal change on T2-weighted sequences (as sometimes seen with longstanding or progressive MS)

Abbreviations: LETM = longitudinally extensive transverse myelitis lesions; $M S=$ multiple sclerosis; NMO = neuromyelitis optica; NMOSD = neuromyelitis optica spectrum disorders. These are some common or key findings that should prompt thorough investigation for competing differential diagnoses before making a diagnosis of NMOSD. associated with acute myelitis is the most specific neuroimaging characteristic of NMOSD and is very uncommon in adult MS. ${ }^{2}$ Such lesions typically involve the central gray matter and may be associated with cord swelling, central hypointensity on T1weighted sequences, and enhancement following IV gadolinium administration; extension of a cervical lesion into the brainstem is characteristic. ${ }^{4,32,33, e 35, \mathrm{e} 64-\mathrm{e} 67}$ In contrast, MS cord lesions are usually about 1 vertebral segment long or less, occupy peripheral white matter tracts such as the dorsal columns, and may be asymptomatic (see table 3 for neuroimaging red flags relevant to MS and other diseases). ${ }^{34-36, e 68-\mathrm{e} 70}$ Although the LETM pattern is characteristic of NMOSD, $7 \%-14 \%$ of initial and $8 \%$ of subsequent myelitis attacks in AQP4-IgG-seropositive patients do not meet the LETM definition. . $^{32,37}$ Therefore, NMOSD must be considered in the differential diagnosis in patients presenting with short myelitis lesions. The timing of an individual MRI scan must be correlated with clinical status. Occasionally, lesions of less than 3 segments are detected in NMOSD because the MRI was performed early in the evolution of acute myelitis or in clinical remission, during which a LETM lesion may fragment into discontinuous lesions. ${ }^{37, e 71}$ Some patients with progressive MS have coalescent cord lesions that can superficially suggest a LETM pattern ${ }^{\mathrm{e} 72}$; both axial and sagittal plane images should be used to judge lesion extent. A LETM MRI pattern may also occur in patients with infectious, granulomatous, neoplastic, and paraneoplastic diseases, acute disseminated encephalomyelitis (ADEM), spinal cord infarction, and dural arteriovenous fistula. ${ }^{\text {ec3 }}$

During optic neuritis attacks, increased signal within the optic nerve may be detected with fatsuppressed T2-weighted orbital MRI sequences, typically with gadolinium enhancement seen on T1weighted sequences (figure 1). Bilateral optic nerve involvement, posterior nerve predominance (especially with extension into the optic chiasm), or extensive lesions of the optic nerve (more than half of its length) are all suggestive of NMOSD (table 3). e $^{e 1, e 73,674}$

According to the 2006 diagnostic scheme, a normal brain MRI or detection of only nonspecific white matter lesions was a key supportive criterion. ${ }^{2}$ Sixty percent of patients with NMOSD accumulate asymptomatic white matter lesions on longitudinal study and as many as $16 \%$ fulfill Barkhof MS MRI criteria. ${ }^{33,38,39, e 75-e 76}$ Detection of brain MRI white matter lesions compatible with MS does not exclude the diagnosis of NMOSD but is considered a red flag, indicating that additional evidence may be required to confidently distinguish NMOSD from MS in individual cases. ${ }^{33,38, e 64, e 76, e 77}$ Analysis of qualitative features of lesions assists in distinguishing NMOSD 


\section{Table 3 Neuroimaging characteristics of NMOSD}

\section{Spinal cord MRI, acute}

LETM lesion associated with acute TM

Increased signal on sagittal T2-weighted (standard T2-weighted, proton density, or STIR sequences) extending over 3 or more complete vertebral segments (figure 1, A and D)

Central cord predominance (more than $70 \%$ of the lesion residing within the central gray matter) (figure 1, A-D)

Gadolinium enhancement of the lesion on T1-weighted sequences (no specific distribution or pattern of enhancement is required) (figure 1, C, E, and F)

Other characteristic features that may be detected

Rostral extension of the lesion into the brainstem (figure 1, D and E)

Cord expansion/swelling

Decreased signal on T1-weighted sequences corresponding to region of increased T2-weighted signal (figure 1F)

Spinal cord MRI, chronic

Longitudinally extensive cord atrophy (sharply demarcated atrophy extending over $\geq 3$ complete, contiguous vertebral segments and caudal to a particular segment of the spinal cord), with or without focal or diffuse T2 signal change involving the atrophic segment (figure 1, $\mathrm{G}$ and $\mathrm{H}$ )

\section{Optic nerve MRI}

Unilateral or bilateral increased T2 signal or T1 gadolinium enhancement within optic nerve or optic chiasm (figure 1, I-K); relatively long lesions (e.g., those extending more than half the distance from orbit to chiasm) and those involving the posterior aspects of the optic nerves or the chiasm are associated with NMO

Cerebral MRI: NMOSD-typical brain lesion patterns (increased signal on T2-weighted MRI sequences unless otherwise noted)

Lesions involving the dorsal medulla (especially the area postrema), either small and localized, often bilateral, or contiguous with an upper cervical spinal cord lesion (figure 2, A-E)

Periependymal surfaces of the fourth ventricle in the brainstem/cerebellum (figure 2, F-H)

Lesions involving the hypothalamus, thalamus, or periependymal surfaces of the third ventricle (figure $3, A$ and $B$ )

Large, confluent, unilateral, or bilateral subcortical or deep white matter lesions (figure 3, C and D)

Long ( $1 / 2$ of the length of the corpus callosum or greater), diffuse, heterogeneous, or edematous corpus callosum lesions (figure $3 \mathrm{E}$ )

Long corticospinal tract lesions, unilateral or bilateral, contiguously involving internal capsule and cerebral peduncle (figure 3F)

Extensive periependymal brain lesions, often with gadolinium enhancement (figure $3, \mathrm{G}-\mathrm{I}$ )

Abbreviations: LETM = longitudinally extensive transverse myelitis lesions; NMOSD = neuromyelitis optica spectrum disorders; STIR = short tau inversion recovery.

from MS. First, at least $7 \%$ of patients have NMOSD-typical patterns that, although not pathognomonic for NMOSD, are considered exceptional for MS (table 3), ${ }^{39}$ including lesions of the dorsal medulla/area postrema (figure 2) 22,33,40,e36,e73,e 78 and periependymal regions in the brainstem (figure 2) $33,40, e 36, \mathrm{e} 73$ and diencephalic structures, ${ }^{39,40, \mathrm{e} 79}$ or cerebral hemispheres ${ }^{\mathrm{e} 80}$ (figure 3), or long lesions spanning much of the length of the corpus callosum ${ }^{39,41}$ or corticospinal tracts (figure 3). ${ }^{33,34}$ Large, confluent, or tumefactive cerebral lesions may suggest NMOSD but in isolation may not be discernable from atypical MS lesions, especially in AQP4-IgG-seronegative patients. ${ }^{27,33,39,42, \mathrm{e} 46, \mathrm{e} 81-\mathrm{e} 83}$ Second, some MRI lesion patterns considered typical of MS are rarely seen in NMOSD, including perpendicular orientation of periventricular lesions (Dawson fingers), periventricular lesions located in the inferior temporal lobe, and cortical lesions. ${ }^{33,38,43, e 64}$ Recent 7T MRI studies revealed differences between NMOSD and MS specifically regarding cortical lesions (frequent in MS but absent in NMOSD) and white matter lesions (MS plaques are periventricular and traversed by a central venule whereas NMOSD lesions are subcortical and lack a central venule). ${ }^{\text {e8,e85 }}$ However, this technology is not widely available and further validation is needed.

The Panel considered evidence for use of other imaging modalities, such as nonconventional MRI techniques and optical coherence tomography, and neurophysiologic tests such as visual evoked potentials. None of these modalities were included in the revised diagnostic criteria because of concerns regarding lack of evidence, specificity, or reliability.

Considerations for AQP4-IgG serologic and other laboratory testing. Technological advances in AQP4IgG assays have improved diagnostic sensitivity without compromising specificity. ${ }^{44}$ The Panel recommended testing with cell-based serum assays (microscopy or flow cytometry-based detection) whenever possible because they optimize autoantibody detection (mean sensitivity $76.7 \%$ in a pooled analysis; $0.1 \%$ false-positive rate in a MS clinic cohort)..$^{12-14,44,45}$ However, cell-based assays are not yet widely available.

Indirect immunofluorescence assays and ELISAs have lower sensitivity (mean sensitivity 63\%-64\% each) and occasionally yield false-positive results $(0.5 \%-1.3 \%$ for ELISA), often at low titer. ${ }^{12-14,44,45, e 86}$ The Panel strongly recommended interpretative caution if such assays are used and when low-titer positive ELISA results are detected in individuals who present with NMOSD clinical symptoms less commonly associated with AQP4IgG (e.g., presentations other than recurrent optic neuritis, myelitis with LETM, or area postrema syndrome) or in situations where clinical evidence suggests a viable alternate diagnosis. Confirmatory testing is recommended, ideally using 1 or more different AQP4$\mathrm{IgG}$ assay techniques. Cell-based assay has the best current sensitivity and specificity and samples may need to be referred to a specialized laboratory.

Patients who fulfill NMOSD criteria but do not have detectable AQP4-IgG despite use of the best available assays, or for whom serologic testing is unavailable, sometimes represent a diagnostic challenge. ${ }^{46}$ A greater proportion of patients with $\mathrm{NMO}$ who have monophasic disease appear to be AQP4-IgG-seronegative compared to those with established relapsing disease. ${ }^{37} \mathrm{~A}$ minority of patients with clinical characteristics of NMO, almost all AQP4IgG-seronegative, have been reported to have detectable serum myelin oligodendrocyte glycoprotein 


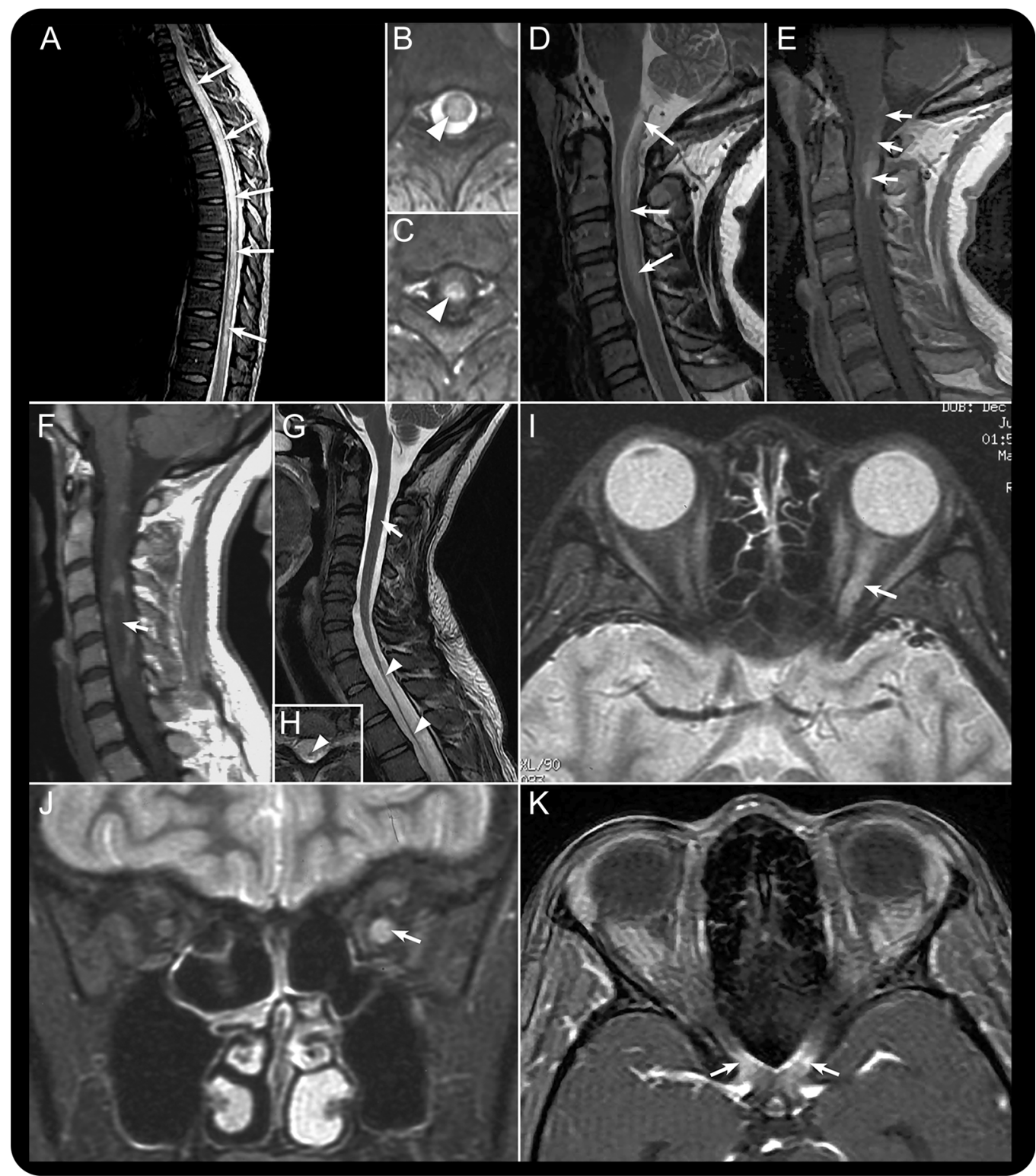

Spinal cord imaging in the context of acute myelitis in neuromyelitis optica spectrum disorders (NMOSD) usually reveals a longitudinally extensive transverse myelitis (LETM) lesion extending over 3 or more vertebral segments. Sagittal T2weighted MRI of the thoracic spinal cord (A) demonstrates a typical LETM lesion involving most of the thoracic spinal cord (arrows). LETM lesions have a predilection for the central cord, as shown by axial T2-weighted (B; arrowhead) and T1weighted MRI with gadolinium ( $C$; arrowhead). Cervical LETM may extend into the medulla, a characteristic NMOSD pattern demonstrated in D (arrows; sagittal T2-weighted MRI) and E (arrows; sagittal T1-weighted MRI with gadolinium). Acute LETM lesions can be associated with intralesional hypointensity as shown by sagittal T1-weighted MRI (F; arrow); in this example, a rim of gadolinium enhancement surrounds the hypointense region. Chronic sequelae of LETM may include longitudinally extensive segments of spinal cord atrophy as shown by T2-weighted MRI using sagittal (G; the 2 arrowheads indicate the atrophic segment and the top arrow indicates the normal diameter of unaffected cervical spinal cord) and axial planes $(\mathrm{H}$; arrowhead shows an atrophic spinal cord). Fast spin echo fat-suppressed T2-weighted MRI in the axial $(\mathrm{I})$ and coronal $(\mathrm{J})$ planes shows increased signal throughout most the length of the left optic nerve, especially its posterior portion (arrows). Axial T1-weighted MRI with gadolinium shows enhancement of the optic chiasm ( $\mathrm{K}$; arrows). These images are from 2 different patients experiencing acute optic neuritis in the setting of NMOSD.

(MOG) antibodies and might have different characteristics (younger age, less frequently female, and less likely to relapse) from those with AQP4-IgG.47-49,e87 These findings might suggest that some AQP4-IgGseronegative patients with clinical and neuroimaging features of NMOSD have a different underlying pathogenesis ${ }^{46}$; the role of MOG or other antibodies in disease pathogenesis remains undetermined. ${ }^{888}$ The IPND nomenclature is designed to allow incorporation of cases of NMOSD associated with other validated biomarkers (e.g., NMOSD with a specific autoantibody). 
Figure 2 Dorsal medulla, area postrema, and other brainstem lesions in neuromyelitis optica spectrum disorder

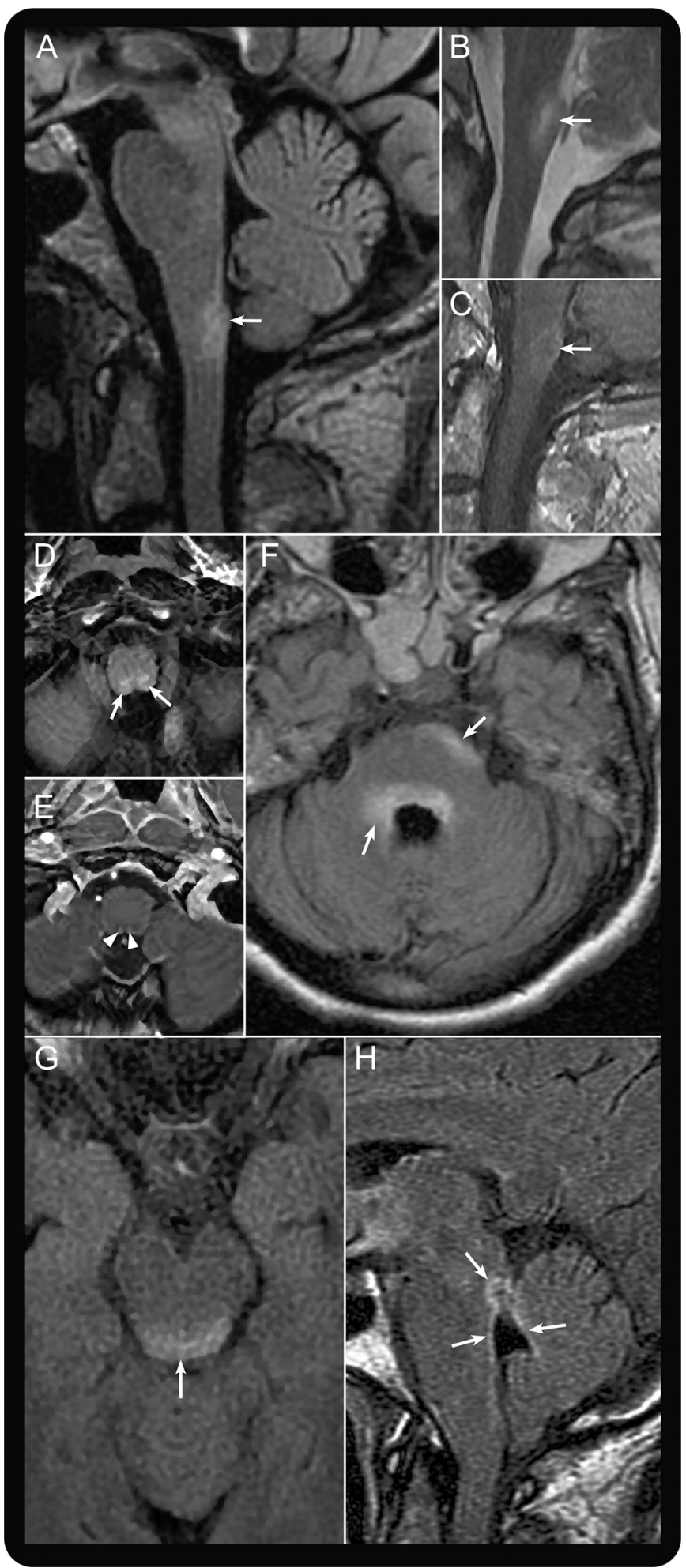

Sagittal T2-weighted fluid-attenuated inversion recovery (FLAIR) MRI shows a lesion in the dorsal medulla ( $\mathrm{A}$; arrow). Sagittal T2-weighted (B) and T1-weighted MRI with gadolinium (C) each demonstrate an acute lesion (arrows) associated with area postrema clinical syndrome. Axial T2-weighted FLAIR ( $D$; arrows) and T1-weighted MRI with gadolinium ( $E_{\text {; }}$ arrowheads) show dorsal medulla involvement in a patient with acute area postrema clinical syndrome. Axial T2-weighted FLAIR MRI shows periependymal lesions involving the pons ( $F$; arrows) and dorsal midbrain (G; arrow). Sagittal T2-weighted FLAIR MRI shows increased signal surrounding the fourth ventricle $(H$; arrows).
Occasionally, patients without detectable serum AQP4-IgG are later found to be seropositive. There may be technical explanations in some cases but antibody levels also increase with clinical relapses and decrease with immunosuppressive therapy in some patients. ${ }^{50}$ Therefore, retesting should be considered before B-cell or antibody-targeted therapies (plasma exchange, immunosuppressive drugs) are instituted and in seronegative patients who relapse. ${ }^{51}$ Cases of clinical NMO in which AQP4-IgG was detected in CSF, but not serum, have been reported but this appears to be rare. ${ }^{52, e 89-e 91}$ Routine CSF testing of AQP4-IgG-seronegative patients is not recommended but might be considered in selected seronegative cases, especially those with additional confounding serum autoantibodies that may lead to uninterpretable or false-positive assay results.

The Panel considered absence of CSF oligoclonal bands as supportive evidence for NMOSD (although they are sometimes transiently detectable at the time of an attack) and presence of bands a red flag, but sensitivity and specificity are modest. ${ }^{4,53}$ CSF pleocytosis $>50$ leukocytes $/ \mu \mathrm{L}$ (incidence approximately $35 \%$ in NMOSD) or the presence of neutrophils or eosinophils (either $>5 / \mu \mathrm{L}$; incidence $44 \%$ and $10 \%$, respectively, in attack-associated samples) are particularly useful in distinguishing NMOSD from MS., ${ }^{4,53}$ CSF glial fibrillary acidic protein also shows promise as a diagnostic and prognostic biomarker but is elevated only for days to weeks following an attack..$^{\text {e92,e93 }}$

Pediatric NMOSD criteria. The Pediatric Working Group members noted that most clinical, neuroimaging, and laboratory characteristics of pediatric NMOSD are similar to those of adult-onset disease. The female preponderance may be of lower magnitude $(\sim 3: 1$ female:male ratio compared with up to $9: 1$ for adults), ${ }^{54, e 94}$ a greater proportion of children may have monophasic disease, ${ }^{54,55, e 95}$ and acute CSF abnormalities in pediatric MS may mimic those ordinarily considered suggestive of NMOSD.54-56 The Working Group identified caveats to application of the adult NMOSD criteria in children, noting especially that detection of a LETM MRI lesion associated with acute myelitis may be less specific for NMOSD. Approximately 15\% of children with MS may have LETM during relapse, LETM can accompany monophasic ADEM, and AQP4-IgG is rarely detected in children with monophasic LETM. $^{55}$ In children diagnosed with ADEM according to international consensus criteria, which require a polyfocal demyelinating clinical presentation with encephalopathy, ${ }^{57}$ the presence of AQP4-IgG favors a diagnosis of NMOSD, although prospective validation studies are required to determine risk of recurrent NMOSD events. In one study of AQP4-IgG-seropositive children, 45\% 

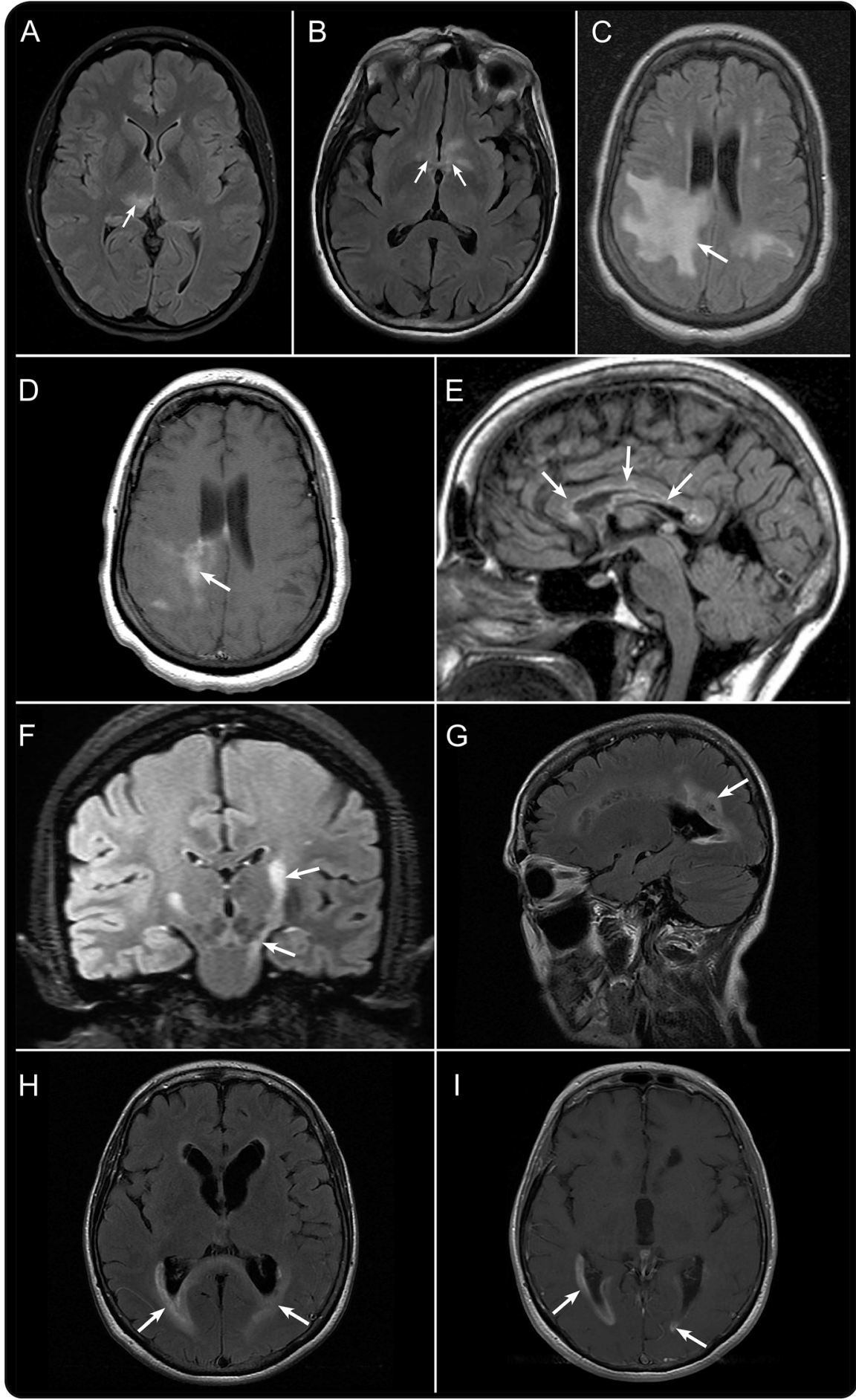

A variety of brain lesion patterns are associated with neuromyelitis optica spectrum disorder. Axial T2-weighted fluidattenuated inversion recovery (FLAIR) MRI from 2 patients demonstrates lesions involving the right thalamus ( $A$; arrow) and the hypothalamus (B; arrows). Axial T2-weighted FLAIR MRI shows an extensive subcortical white matter lesion ( $C$; arrow) that enhances after gadolinium administration on T1-weighted sequences ( $D$; arrow). Chronic longitudinally extensive and linear corpus callosum lesions are depicted on sagittal T2-weighted FLAIR MRI ( $E_{;}$arrows). Coronal T2-weighted FLAIR MRI shows longitudinal involvement of the corticospinal tract extending to the cerebral peduncle and pons ( $F$; arrows). Acute periependymal cerebral lesions from one patient are depicted using sagittal (G; arrow) and axial ( $\mathrm{H}$; arrows) T2-weighted FLAIR MRI and axial T1-weighted MRI with gadolinium (I; arrows). 
experienced recurrent cerebral manifestations including encephalopathy. ${ }^{56}$ Aside from these caveats, the currently proposed diagnostic criteria for NMOSD are appropriate for pediatric patients. Longitudinal observation of the clinical course for dissemination in time and retesting the AQP4-IgG status of some children, especially AQP4-IgG-seronegative individuals presenting with an ADEM-like event that includes optic neuritis and LETM, may be required to achieve confident diagnosis.

Monophasic NMOSD. The occurrence of a second clinical attack, which defines a relapsing disorder, was often considered sufficient to revise the diagnosis to MS in otherwise typical NMO cases. Approximately $5 \%-10 \%$ of contemporary cases are described as monophasic, although the optimal definition for monophasic NMOSD remains elusive. It is unclear whether the occurrence of bilateral optic neuritis and myelitis at initial presentation are helpful or essential to distinguish such cases from relapsing NMOSD. Similarly, the interval between index clinical events that is compatible with monophasic NMO has not been consistently defined or adequately examined as a predictor of future clinical course. Although early risk of relapse is high in AQP4-IgG-seropositive cases (e.g., about 60\% within 1 year after LETM), ${ }^{20}$ cases have been documented in whom more than a decade elapsed between the index events and relapse. ${ }^{\mathrm{e} 4}$

Several studies show a pattern of apparently monophasic NMO being associated with a more equitable sex distribution, relatively younger age at disease onset, tendency to present with simultaneous myelitis and bilateral optic neuritis (rather than unilateral optic nerve involvement), lower frequency of other autoimmune diseases, and lower prevalence of serum AQP4-IgG compared to relapsing NMO.4,37,e96 A fraction of these patients may have other serum antibodies such as MOG-IgG. ${ }^{47}$

The Panel concluded that monophasic NMOSD is a recognizable clinical entity but that criteria that accurately predict long-term adherence to a monophasic course cannot currently be defined. An interval longer than 4 weeks between index attacks indicates relapsing disease. The Panel also recommended that at least 5 years (preferably longer) of relapse-free clinical observation after the index events be required before a monophasic course is assumed with any degree of confidence. Patients who are AQP4-IgGseropositive should be assumed to be at risk for relapse indefinitely and preventive treatment should be considered, even in the setting of a prolonged clinical remission.

Systemic autoimmunity associated with NMOSD. Based on evidence from several observational studies, the
Panel concluded that clinical diagnoses of SLE, SS, or myasthenia gravis may coexist with NMOSD clinical syndromes in AQP4-IgG-seropositive patients and, in fact, their presence strengthens confidence about a NMOSD diagnosis. ${ }^{58, \mathrm{e} 97, \mathrm{e} 98}$ The underlying cause of CNS symptoms and signs is more likely to be co-associated NMOSD than a direct complication (e.g., associated vasculitis) of SLE or SS. In a patient suspected of having NMOSD, the presence of clinical myasthenia gravis or detectable serum acetylcholine receptor antibodies is considered supportive of NMOSD diagnosis. ${ }^{\text {e99-e101 }}$

Pathology. Pathologic findings in biopsy or autopsy tissue obtained from patients with AQP4-IgG-seropositive NMOSD demonstrate loss of AQP4 immunoreactivity and evidence of perivascular complement activation in actively demyelinating lesions. ${ }^{\text {e20,e21 }}$ Additionally, findings supportive of astrocytopathy such as truncated astrocyte processes or cell loss may be detected by immunostaining for glial fibrillary acidic protein. ${ }^{59, \mathrm{e} 20}$ These findings in active lesions distinguish AQP4-IgGpositive NMOSD from MS; data from seronegative individuals are not yet available. ${ }^{59}$ Necrosis and lesion infiltration with neutrophils and eosinophils are supportive characteristics, ${ }^{\text {e102 }}$ but may not be present. Spinal cord lesions may differ from supraspinal lesions in this respect. ${ }^{\text {e103 }}$ Serum AQP4-IgG testing usually obviates the need for biopsy in severe myelitis and leukoencephalopathy syndromes. ${ }^{\text {e19,e104,e105 }}$ Aquaporin-4 immunostaining is not a routine procedure in CNS biopsy processing and is available only in select centers. The Panel does not recommend CNS biopsy but recognizes that in atypical cases, expert pathologic review of biopsy tissue of brain or spinal cord might help establish NMOSD and exclude competing diagnoses. ${ }^{60}$

Opticospinal MS. The term opticospinal MS was introduced in Japan to refer to a pattern of MS that is relatively more common in Asian countries. ${ }^{11, \mathrm{e} 106}$ It was defined by recurrent optic neuritis and myelitis attacks with no brain involvement, although some investigators allow occurrence of certain brainstem syndromes. ${ }^{\text {e107 }}$ The description of opticospinal MS represented an important milestone because it recognized that there was a relapsing illness distinct from conventional MS that selectively targeted the optic nerve and spinal cord at a time when relapses were deemed to be incompatible with a diagnosis of NMO in both Western countries and Japan.

Contemporary NMO criteria that include MRI criteria for length of spinal cord lesions and detection of AQP4-IgG are more specific than historical opticospinal MS criteria. Clinicians and investigators in many Asian countries now use the term NMO rather 
than opticospinal MS in research and practice. When similarly defined in Asia, NMO clinical syndromes and brain MRI lesions are both analogous to those encountered in Western countries. 22,23,33,39,e108 The Panel concluded that opticospinal MS is a historically important but now superseded term, especially in light of the need to distinguish NMO from MS to guide treatment decisions. ${ }^{15-18, \mathrm{e} 24-\mathrm{e} 26}$

DISCUSSION The high specificity of AQP4-IgG has been exploited to expand the clinical and neuroimaging spectrum of NMO. The consensus definition of NMOSD unifies traditional NMO and modern NMOSD definitions. It allows for NMOSD diagnosis in AQP4-IgG-seropositive patients with involvement of almost any CNS region as well as in those with restricted involvement of a single region (e.g., recurrent transverse myelitis). For the first time, criteria allow for NMOSD diagnosis in patients who have not experienced clinical involvement of either optic nerves or spinal cord. The Panel reached these conclusions for the following reasons: (1) there are no established biological differences between patients diagnosed with NMO compared with NMOSD (using 2006 and 2007 definitions, respectively) in AQP4-IgG-seropositive patients; (2) limited NMOSD syndromes affecting CNS regions other than the optic nerve and spinal cord often herald subsequent clinical attacks consistent with conventional $\mathrm{NMO}$ in AQP4-IgG-positive patients; and (3) current immunotherapeutic strategies are the same for relapsing NMO and NMOSD, regardless of AQP4-IgG serologic status. To enhance criteria specificity, AQP4-IgG-seronegative patients must have experienced at least one of the 3 most common clinical characteristics of seropositive NMOSD, namely optic neuritis, transverse myelitis with LETM, or area postrema syndrome with associated MRI lesions. These criteria are appropriate for adults and, with minor caveats, children. Finally, the Panel cautions against making a diagnosis of monophasic NMOSD, especially in AQP4-IgG-seropositive patients, and recommends abandonment of the term opticospinal MS for cases that meet NMOSD criteria.

The IPND criteria are expected to facilitate earlier and more accurate diagnosis by identifying individuals who would have been diagnosed with idiopathic transverse myelitis, idiopathic optic neuritis, or atypical MS. This will be particularly true for AQP4-IgGseropositive patients experiencing their first CNS attack (such patients will now meet NMOSD criteria), leading to a specific treatment path with immunotherapy for attack prevention. The criteria should also provide greater specificity for distinguishing both AQP4-IgG-seropositive and AQP4-IgG-seronegative NMOSD from MS. Early-stage diagnostic specificity is critical because recent observational data suggest that interferon- $\beta$, natalizumab, and fingolimod may worsen NMO. ${ }^{15-18, e 24-226}$

The IPND criteria are expected to facilitate more comprehensive and comparable epidemiologic studies by supplying a uniform case definition and a glossary of defined terms. For AQP4-IgG-seronegative cases diagnosed using the new NMOSD scheme, detailed clinical, neuroimaging, and laboratory descriptions of patients will be necessary to better characterize this heterogeneous population. This is particularly important to identify the frequencies with which seroconversion to AQP4-IgG positivity or detection of other autoantibodies of interest occur and to identify phenocopies later diagnosed as other conditions.

Many observations used to construct the IPND criteria were derived from relatively small case series. The Panel recommends several large-scale prospective validation and assessment strategies. These include but are not limited to studies that (1) systematically apply the criteria to consecutive cases of NMOSD, with and without $\mathrm{AQP} 4-\mathrm{IgG}$, to determine whether the diagnosis is confirmed or another alternative diagnosis emerges in follow-up; (2) validate the association and potential immunopathogenesis of serum MOG-IgG (and other future potential laboratory and neuroimaging biomarkers) with clinically diagnosed NMOSD; (3) identify clinical settings in which false-negative and false-positive AQP4-IgG results are more likely; (4) validate different definitions predictive of clinical course (monophasic vs relapsing) and determine the distinguishing characteristics of these subtypes; and (5) further examine the immunopathology of NMOSD to determine whether there is unrecognized heterogeneity to inform its classification, especially of AQP4-IgG-seronegative cases. These and other approaches to criteria validation will allow future refinement of NMOSD diagnostic criteria commensurate with the next era of scientific advances.

\section{AUTHOR CONTRIBUTIONS}

D. Wingerchuk participated in study concept and design, obtaining funding, acquisition and analysis of data, drafting and revising the manuscript, and coordinating the study. B. Banwell participated in the acquisition and analysis of data and writing and critical review of the manuscript for important intellectual content. J. Bennett participated in the acquisition and analysis of data and writing and critical review of the manuscript for important intellectual content. P. Cabre participated in the acquisition and analysis of data and writing and critical review of the manuscript for important intellectual content. W. Carroll participated in the acquisition and analysis of data and writing and critical review of the manuscript for important intellectual content. T. Chitnis participated in the acquisition and analysis of data and writing and critical review of the manuscript for important intellectual content. J. de Seze participated in the acquisition and analysis of data and writing and critical review of the manuscript for important intellectual content. K. Fujihara participated in the acquisition and analysis of data and writing and critical review of the manuscript for important intellectual content. B. Greenberg participated in the acquisition and analysis of data and writing and critical 
review of the manuscript for important intellectual content. A. Jacob participated in the acquisition and analysis of data and writing and critical review of the manuscript for important intellectual content. S. Jarius participated in the acquisition and analysis of data and writing and critical review of the manuscript for important intellectual content. M. Lana-Peixoto participated in the acquisition and analysis of data and writing and critical review of the manuscript for important intellectual content. M. Levy participated in the acquisition and analysis of data and writing and critical review of the manuscript for important intellectual content. J. Simon participated in the acquisition and analysis of data and writing and critical review of the manuscript for important intellectual content. S. Tenembaum participated in the acquisition and analysis of data and writing and critical review of the manuscript for important intellectual content. A. Traboulsee participated in the acquisition and analysis of data and writing and critical review of the manuscript for important intellectual content. P. Waters participated in the acquisition and analysis of data and writing and critical review of the manuscrip for important intellectual content. K. Wellik participated in the acquisition and analysis of data and writing and critical review of the manuscript for important intellectual content. B. Weinshenker participated in study concept and design, obtaining funding, acquisition and analysis of data, drafting and revising the manuscript, and coordinating the study.

\section{ACKNOWLEDGMENT}

The authors thank the Guthy-Jackson Charitable Foundation for organizational and financial support; Dr. Katja van Herle, Dr. Valerie Pasquetto, Dr. Michael Yeaman, and Jacinta Behne for assistance; Prof. Jun-ichi Kira, Kyushu University, for initial IPND discussions; and Amy Clayton and Katrina Rivera for administrative assistance and Cory Poetzl and Bonnie Schimek for media support services (Mayo Clinic, Scottsdale, AZ).

\section{STUDY FUNDING}

The International Panel for Neuromyelitis Optica Diagnosis was supported by the Guthy Jackson Charitable Foundation.

\section{DISCLOSURE}

D. Wingerchuk has received research support from Alexion, TerumoBCT, and the Guthy Jackson Charitable Foundation, receives financial compensation for participation on a relapse adjudication panel for MedImmune, has served as a consultant to Alexion, MedImmune, and Chugai Pharmaceuticals, and has served as co-Editor-in-Chief of The Neurologist. B. Banwell serves as an advisor on clinical trial design for Biogen Idec, Sanofi, Teva Neuroscience, and Novartis. Dr. Banwell provides consultant services for Novartis. Dr. Banwell is an Editor-in-Chief for Multiple Sclerosis and Related Disorders. J. Bennett serves as a consultant for Novartis Pharmaceuticals, Alnaylam Pharmaceuticals, MedImmune, Chugai Pharmaceuticals, EMD Serono, Abbott Pharmaceuticals, Genentech, Genzyme, and Questcor Pharmaceuticals; receives license royalties for a patent re: Compositions and Methods for the Treatment of Neuromyelitis Optica; and serves on the editorial boards of the Multiple Sclerosis Journal and Journal of Neuroophthalmology. P. Cabre reports no disclosures relevant to the manuscript. W. Carroll has received travel assistance and/or honoraria from, and provided advice to, Bayer Schering Pharma, Biogen Idec, Novartis, Genzyme, SanofiAventis, CSL, and Merck-Serono. T. Chitnis has performed consulting for Biogen Idec and Novartis. Has received support for research activities from Novartis and Merck-Serono. J. de Seze has performed consulting for Alexion, Chugai, and Novartis. K. Fujihara serves on scientific advisory boards for Bayer Schering Pharma, Biogen Idec, Mitsubishi Tanabe Pharma Corporation, Novartis Pharma, Chugai Pharmaceutical, Ono Pharmaceutical, Nihon Pharmaceutical, Merck Serono, Alexion Pharmaceuticals, Medimmune, and Medical Review; has received funding for travel and speaker honoraria from Bayer Schering Pharma, Biogen Idec, Eisai Inc., Mitsubishi Tanabe Pharma Corporation, Novartis Pharma, Astellas Pharma Inc., Takeda Pharmaceutical Company Limited, Asahi Kasei Medical Co., Daiichi Sankyo, and Nihon Pharmaceutical; serves as an editorial board member of Clinical and Experimental Neuroimmunology (2009-present) and an advisory board member of Sri Lanka Journal of Neurology; has received research support from Bayer Schering Pharma, Biogen Idec Japan, Asahi Kasei Medical, The ChemoSero-Therapeutic Research Institute, Teva Pharmaceutical, Mitsubishi Tanabe Pharma, Teijin Pharma, Chugai Pharmaceutical, Ono Pharmaceutical,
Nihon Pharmaceutical, and Genzyme Japan; and is funded as the secondary investigator (22229008, 2010-2015) by the Grants-in-Aid for Scientific Research from the Ministry of Education, Science and Technology of Japan and as the secondary investigator by the Grants-in-Aid for Scientific Research from the Ministry of Health, Welfare and Labor of Japan (2010-present). B. Greenberg has received compensation for consulting services from Chugai, GSK, Biogen, Novartis, DioGenix, and Amplimmune and receives research support from the Guthy Jackson Charitable Foundation. A. Jacob has received honoraria for clinical trial advisory boards and speaker's fees from Chugai, Alexion, and Biogen. S. Jarius has been supported by a research grant from the European Committee for Treatment and Research in Multiple Sclerosis (ECTRIMS); S. Jarius' department has been supported by research grants from Merck Serono and from the Dietmar Hopp Foundation and by a travel grant from the Guthy-Jackson Charitable Foundation. M. Lana-Peixoto has served on a scientific advisory board for Novartis. M. Levy is supported by research funds from the NIH, Guthy Jackson Charitable Foundation, Viropharma, Acorda, ApoPharma, and Sanofi. He has received advisory compensation from GlaxoSmithKline and Asterias. J. Simon has served as a consultant and received research support from Biogen Idec. S. Tenembaum has received honoraria for speaking from Biogen Idec and Merck Serono and for participation in an advisory group for Biogen Idec, Merck Serono, and Teva Pharmaceutical Ind. A. Traboulsee has received grant funding from the MS Society of Canada, the Canadian Institute for Health Research, the Lotte and John Hecht Foundation, the Vancouver Hospital Foundation, Bayer, Roche, and Biogen. He has served on the data safety monitoring board for Merck Serono and a clinical trial steering committee for Roche. He has received honoraria or travel grants from Biogen, Teva Canada Innovation, Roche, Merck/EMD Serono, and Chugai Pharmaceuticals. P. Waters is a named inventor on patents for antibody assays (no. 20120114666, application no. 1310855.0) and has received royalties. He has received a speaker honorarium from Biogen Idec and Euroimmun AG. K. Wellik reports no disclosures relevant to the manuscript. B. Weinshenker has received a research grant from the Guthy Jackson Foundation. He receives royalties from RSR, Ltd. for a technology license related to a test for aquaporin- 4 autoantibodies for diagnosis of neuromyelitis optica. $\mathrm{He}$ receives financial compensation for service on data safety monitoring committees for Novartis, Biogen Idec, and Mitsubishi pharmaceutical companies for MS clinical trials, and on a relapse adjudication panel for MedImmune Pharmaceuticals for NMO clinical trials. He served as a consultant for GlaxoSmithKline, Elan, Ono, Chugai, Chord, and Alexion pharmaceutical companies and for Asahi Kasei Medical Company, all for activities related to design of clinical trials for neuromyelitis optica. He serves on editorial boards for Neurology ${ }^{\circledR}$, the Canadian Journal of Neurological Sciences, and the Turkish Journal of Neurology. Go to Neurology.org for full disclosures.

Received September 30, 2014. Accepted in final form January 28, 2015.

\section{REFERENCES}

1. Wingerchuk DM, Lennon VA, Lucchinetti CF, Pittock SJ, Weinshenker BG. The spectrum of neuromyelitis optica. Lancet Neurol 2007;6:805-815.

2. Wingerchuk DM, Lennon VA, Pittock SJ, Lucchinetti CF, Weinshenker BG. Revised diagnostic criteria for neuromyelitis optica. Neurology 2006;66:1485-1489.

3. Jarius $S$, Wildemann B. The history of neuromyelitis optica. J Neuroinflammation 2013;10:8.

4. Wingerchuk DM, Hogancamp WF, O'Brien PC, Weinshenker BG. The clinical course of neuromyelitis optica (Devic's syndrome). Neurology 1999;53:1107-1114.

5. Lennon VA, Wingerchuk DM, Kryzer TJ, et al. A serum autoantibody marker of neuromyelitis optica: distinction from multiple sclerosis. Lancet 2004;364:2106-2112.

6. Lennon VA, Kryzer TJ, Pittock SJ, Verkman AS, Hinson SR. IgG marker of optic-spinal multiple sclerosis binds to the aquaporin- 4 water channel. J Exp Med 2005; 202:473-477.

7. Papadopoulos MC, Verkman AS. Aquaporin 4 and neuromyelitis optica. Lancet Neurol 2012;11:535-544. 
8. Asgari N, Lillevang ST, Skejoe HP, Falah M, Stenager E, Kyvik KO. A population-based study of neuromyelitis optica in Caucasians. Neurology 2011;76:1589-1595.

9. Cabre P. Migration and multiple sclerosis: the French West Indies experience. J Neurol Sci 2007;262:117-121.

10. Cabrera-Gomez JA, Kurtzke JF, Gonzalez-Quevedo A, Lara-Rodriguez R. An epidemiological study of neuromyelitis optica in Cuba. J Neurol 2009;256:35-44.

11. Kuroiwa Y, Shibasaki H. Epidemiologic and clinical studies of multiple sclerosis in Japan. Neurology 1976;26:8-10.

12. Jarius S, Wildemann B. Aquaporin-4 antibodies (NMO$\mathrm{IgG}$ ) as a serological marker of neuromyelitis optica: a critical review of the literature. Brain Pathol 2013;23:661-683.

13. Waters PJ, McKeon A, Leite MI, et al. Serologic diagnosis of NMO: a multicenter comparison of aquaporin-4-IgG assays. Neurology 2012;78:665-671.

14. Sato DK, Nakashima I, Takahashi T, et al. Aquaporin-4 antibody-positive cases beyond current diagnostic criteria for NMO spectrum disorders. Neurology 2013;80:2210-2216.

15. Kimbrough DJ, Fujihara K, Jacob A, et al. Treatment of neuromyelitis optica: review and recommendations. Mult Scler Relat Disord 2012;1:180-187.

16. Kleiter I, Hellwig K, Berthele A, et al. Failure of natalizumab to prevent relapses in neuromyelitis optica. Arch Neurol 2012;69:239-245.

17. Min JH, Kim BJ, Lee KH. Development of extensive brain lesions following fingolimod (FTY720) treatment in a patient with neuromyelitis optica spectrum disorder. Mult Scler 2012;18:113-115.

18. Papeix C, Vidal JS, de Seze J, et al. Immunosuppressive therapy is more effective than interferon in neuromyelitis optica. Mult Scler 2007;13:256-259.

19. Matiello M, Lennon VA, Jacob A, et al. NMO-IgG predicts the outcome of recurrent optic neuritis. Neurology 2008;70:2197-2200.

20. Weinshenker BG, Wingerchuk DM, Vukusic S, et al. Neuromyelitis optica IgG predicts relapse after longitudinally extensive transverse myelitis. Ann Neurol 2006;59: 566-569.

21. Nagaishi A, Takagi M, Umemura A, et al. Clinical features of neuromyelitis optica in a large Japanese cohort: comparison between phenotypes. J Neurol Neurosurg Psychiatry 2011;82:1360-1364.

22. Misu T, Fujihara K, Nakashima I, Sato S, Itoyama Y Intractable hiccup and nausea with periaqueductal lesions in neuromyelitis optica. Neurology 2005;65:1479-1482.

23. Apiwattanakul M, Popescu BF, Matiello M, et al. Intractable vomiting as the initial presentation of neuromyelitis optica. Ann Neurol 2010;68:757-761.

24. Kremer L, Mealy M, Jacob A, et al. Brainstem manifestations in neuromyelitis optica: a multicenter study of 258 patients. Mult Scler 2014;20:843-847.

25. Poppe AY, Lapierre Y, Melancon D, et al. Neuromyelitis optica with hypothalamic involvement. Mult Scler 2005; 11:617-621.

26. Vernant JC, Cabre P, Smadja D, et al. Recurrent optic neuromyelitis with endocrinopathies: a new syndrome. Neurology 1997;48:58-64.

27. Kim W, Kim SH, Lee SH, Li XF, Kim HJ. Brain abnormalities as an initial manifestation of neuromyelitis optica spectrum disorder. Mult Scler 2011;17:1107-1112.

28. Magana SM, Matiello M, Pittock SJ, et al. Posterior reversible encephalopathy syndrome in neuromyelitis optica spectrum disorders. Neurology 2009;72:712-717.
29. Kim SM, Go MJ, Sung JJ, Park KS, Lee KW. Painful tonic spasm in neuromyelitis optica: incidence, diagnostic utility, and clinical characteristics. Arch Neurol 2012;69: 1026-1031.

30. Popescu BF, Lennon VA, Parisi JE, et al. Neuromyelitis optica unique area postrema lesions: nausea, vomiting, and pathogenic implications. Neurology 2011;76:1229-1237.

31. Wingerchuk DM, Pittock SJ, Lucchinetti CF, Lennon VA, Weinshenker BG. A secondary progressive clinical course is uncommon in neuromyelitis optica. Neurology 2007;68: 603-605.

32. Flanagan EP, Weinshenker BG, Krecke KN, et al. Short myelitis lesions in aquaporin-4-IgG-positive neuromyelitis optica spectrum disorders. JAMA Neurol 2015;72:81-87.

33. Kim W, Park MS, Lee SH, et al. Characteristic brain magnetic resonance imaging abnormalities in central nervous system aquaporin-4 autoimmunity. Mult Scler 2010; 16:1229-1236

34. Huh SY, Min JH, Kim W, et al. The usefulness of brain MRI at onset in the differentiation of multiple sclerosis and seropositive neuromyelitis optica spectrum disorders. Mult Scler 2014;20:695-704.

35. Bourre B, Zephir H, Ongagna JC, et al. Long-term followup of acute partial transverse myelitis. Arch Neurol 2012; 69:357-362.

36. Scott TF, Kassab SL, Pittock SJ. Neuromyelitis optica IgG status in acute partial transverse myelitis. Arch Neurol 2006;63:1398-1400.

37. Jarius S, Ruprecht K, Wildemann B, et al. Contrasting disease patterns in seropositive and seronegative neuromyelitis optica: a multicentre study of 175 patients. J Neuroinflammation 2012;9:14

38. Matthews L, Marasco R, Jenkinson M, et al. Distinction of seropositive NMO spectrum disorder and MS brain lesion distribution. Neurology 2013;80:1330-1337.

39. Pittock SJ, Lennon VA, Krecke K, Wingerchuk DM, Lucchinetti CF, Weinshenker BG. Brain abnormalities in neuromyelitis optica. Arch Neurol 2006;63:390-396.

40. Pittock SJ, Weinshenker BG, Lucchinetti CF, Wingerchuk DM, Corboy JR, Lennon VA. Neuromyelitis optica brain lesions localized at sites of high aquaporin 4 expression. Arch Neurol 2006;63:964-968.

41. Nakamura M, Misu T, Fujihara K, et al. Occurrence of acute large and edematous callosal lesions in neuromyelitis optica. Mult Scler 2009;15:695-700.

42. Magana SM, Pittock SJ, Lennon VA, Keegan BM, Weinshenker BG, Lucchinetti CF. Neuromyelitis optica IgG serostatus in fulminant central nervous system inflammatory demyelinating disease. Arch Neurol 2009; 66:964-966.

43. Kim JE, Kim SM, Ahn SW, et al. Brain abnormalities in neuromyelitis optica. J Neurol Sci 2011;302:43-48.

44. Waters P, Pittock SJ, Bennett JL, Jarius S, Weinshenker BG, Wingerchuk DM. Evaluation of aquaporin-4 antibody assays. Clin Exp Neuroimmunol 2014;5;290-303.

45. Pittock SJ, Lennon VA, Bakshi N, et al. Seroprevalence of aquaporin-4-IgG in a northern California population representative cohort of multiple sclerosis. JAMA Neurol 2014;71:1433-1436.

46. Marignier R, Bernard-Valnet $\mathrm{R}$, Giraudon $\mathrm{P}$, et al. Aquaporin-4 antibody-negative neuromyelitis optica: distinct assay sensitivity-dependent entity. Neurology 2013; 80:2194-2200 
47. Kitley J, Woodhall M, Waters P, et al. Myelin-oligodendrocyte glycoprotein antibodies in adults with a neuromyelitis optica phenotype. Neurology 2012;79:1273-1277.

48. Kitley J, Waters P, Woodhall M, et al. Neuromyelitis optica spectrum disorders with aquaporin- 4 and myelinoligodendrocyte glycoprotein antibodies: a comparative study. JAMA Neurol 2014;71:276-283.

49. Sato DK, Callegaro D, Lana-Peixoto MA, et al. Distinction between MOG antibody-positive and AQP4 antibody-positive NMO spectrum disorders. Neurology 2014;82:474-481.

50. Jarius S, Aboul-Enein F, Waters P, et al. Antibody to aquaporin- 4 in the long-term course of neuromyelitis optica. Brain 2008;131:3072-3080.

51. Jiao Y, Fryer JP, Lennon VA, et al. Updated estimate of AQP4-IgG serostatus and disability outcome in neuromyelitis optica. Neurology 2013;81:1197-1204.

52. McKeon A, Pittock SJ, Lennon VA. CSF complements serum for evaluating paraneoplastic antibodies and NMO-IgG. Neurology 2011;76:1108-1110.

53. Jarius S, Paul F, Franciotta D, et al. Cerebrospinal fluid findings in aquaporin- 4 antibody positive neuromyelitis optica: results from 211 lumbar punctures. J Neurol Sci 2011;306:82-90.
54. Collongues N, Marignier R, Zephir H, et al. Long-term follow-up of neuromyelitis optica with a pediatric onset. Neurology 2010;75:1084-1088.

55. Banwell B, Tenembaum S, Lennon VA, et al. Neuromyelitis optica-IgG in childhood inflammatory demyelinating CNS disorders. Neurology 2008;70:344-352.

56. McKeon A, Lennon VA, Lotze T, et al. CNS aquaporin- 4 autoimmunity in children. Neurology 2008;71:93-100.

57. Krupp LB, Tardieu M, Amato MP, et al. International Pediatric Multiple Sclerosis Study Group criteria for pediatric multiple sclerosis and immune-mediated central nervous system demyelinating disorders: revisions to the 2007 definitions. Mult Scler 2013;19:1261-1267.

58. Wingerchuk DM, Weinshenker BG. The emerging relationship between neuromyelitis optica and systemic rheumatologic autoimmune disease. Mult Scler 2012;18:5-10.

59. Lucchinetti CF, Guo Y, Popescu BF, Fujihara K, Itoyama $\mathrm{Y}$, Misu T. The pathology of an autoimmune astrocytopathy: lessons learned from neuromyelitis optica. Brain Pathol 2014;24:83-97.

60. Popescu BFG, Guo Y, Jentoft ME, et al. Diagnostic utility of aquaporin- 4 in the analysis of active demyelinating lesions. Neurology 2015;84:148-158. 


\section{Neurology}

International consensus diagnostic criteria for neuromyelitis optica spectrum disorders Dean M. Wingerchuk, Brenda Banwell, Jeffrey L. Bennett, et al.

Neurology 2015;85;177-189 Published Online before print June 19, 2015

DOI 10.1212/WNL.0000000000001729

This information is current as of June 19, 2015

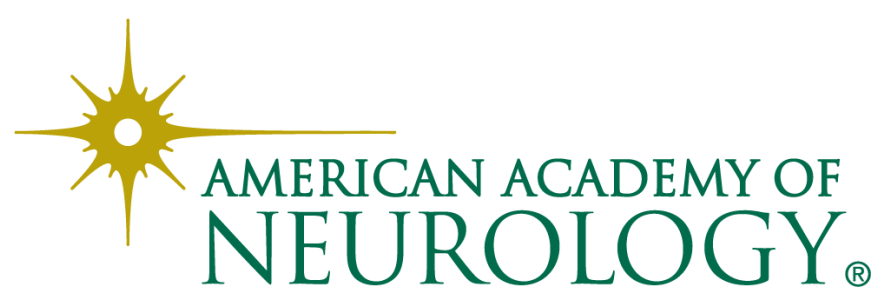




\section{Updated Information \& Services}

\section{Supplementary Material}

\section{References}

\section{Citations}

Subspecialty Collections

\section{Permissions \& Licensing}

\section{Reprints}

including high resolution figures, can be found at: http://n.neurology.org/content/85/2/177.full

Supplementary material can be found at: http://n.neurology.org/content/suppl/2015/06/19/WNL.0000000000001 729.DC1

http://n.neurology.org/content/suppl/2015/06/19/WNL.0000000000001 729.DC2

http://n.neurology.org/content/suppl/2016/04/10/WNL.0000000000001 729.DC3

This article cites 60 articles, 23 of which you can access for free at: http://n.neurology.org/content/85/2/177.full\#ref-list-1

This article has been cited by 79 HighWire-hosted articles: http://n.neurology.org/content/85/2/177.full\#\#otherarticles

This article, along with others on similar topics, appears in the following collection(s):

All Demyelinating disease (CNS)

http://n.neurology.org/cgi/collection/all_demyelinating_disease_cns Devic's syndrome

http://n.neurology.org/cgi/collection/devics_syndrome Multiple sclerosis

http://n.neurology.org/cgi/collection/multiple_sclerosis

Optic neuritis; see Neuro-ophthalmology/Optic Nerve http://n.neurology.org/cgi/collection/optic_neuritis

Transverse myelitis

http://n.neurology.org/cgi/collection/transverse_myelitis

Information about reproducing this article in parts (figures,tables) or in its entirety can be found online at:

http://www.neurology.org/about/about_the_journal\#permissions

Information about ordering reprints can be found online:

http://n.neurology.org/subscribers/advertise

Neurology ${ }^{\circledR}$ is the official journal of the American Academy of Neurology. Published continuously since 1951, it is now a weekly with 48 issues per year. Copyright @ 2015 American Academy of Neurology. All rights reserved. Print ISSN: 0028-3878. Online ISSN: 1526-632X.

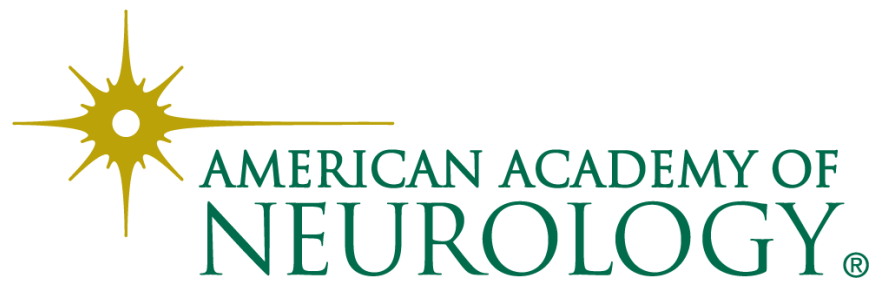

